\title{
Radio Halo and Relic Sources in Galaxy Clusters
}

\author{
Kinwah $\mathrm{Wu}$ \\ Mullard Space Science Laboratory, University College London, \\ Holmbury St Mary, Surrey RH5 6NT, United Kingdom \\ Melanie Johnston-Hollitt \\ Leiden Observatory, P.O. Box 9513, NL-2300 RA, Leiden, The \\ Netherlands
}

Richard Hunstead

School of Physics, University of Sydney, NSW 2006, Australia

\begin{abstract}
We review briefly the general properties of halo and relic sources in galaxy clusters. We also present a polarized radiative transfer algorithm which is useful for calculations of polarized radio emission from halos, relics and active galaxies in a magnetized intra-cluster medium.
\end{abstract}

\section{Introduction}

Radio halos and relics are diffuse sources which appear to be peculiar to galaxy clusters. They are generally believed to be associated with the clusters themselves rather than with individual active galaxies. Typically they have low surface brightness and steep radio spectra, indicating the emission is non-thermal and optically thin. Halos are spatially extended, with sizes of $\sim 500 \mathrm{kpc}$, comparable with the cores of galaxy clusters. They tend to have a round morphology, show little or no polarization, and permeate the cluster centers. Relics are also spatially extended, but they are elongated and are usually located at the cluster periphery. In contrast to halos, emission from relic sources is strongly polarized. (For a review of radio halos and relics, see e.g. Giovannini \& Feretti 2002a). Both radio halos and relics are rare, currently being detected in only about $5 \%$ of clusters. However, the detection rate increases with cluster mass and among the clusters with X-ray luminosity of $10^{45} \mathrm{erg} \mathrm{s}^{-1}$ or above, $1 / 3$ show radio halos or relics (Giovannini \& Feretti 2002b).

Several important aspects of these cluster radio sources were found recently. First, there is a linear relation in the point-to-point spatial distribution of radio and X-ray emission of halos (Govoni et al. 2001). Second, the radio power of relic sources at $1.4 \mathrm{GHz}$ increases with the X-ray temperature of the cluster (Colafrancesco 1999; Liang et al. 2000). Third, the radio power of halos at 1.4 $\mathrm{GHz}$ is correlated with the cluster dipole power ratio (Buote 2001). The first two findings indicate a close connection between the non-thermal electrons for radio synchrotron radiation and the thermal electrons that emit keV X-rays. They also suggest that more massive clusters are more powerful radio sources. The 
dipole power ratio, which is a measure of the degree of departure of a cluster from its virial state, can be caused by core disruption when a violent merging event occurs. The third finding thus implies that the formation of radio halos is a consequence of cluster merging.

A possible scenario is that cluster merging leads to the formation of shocks and turbulence, which in turn accelerate (and/or reaccelerate) electrons to ultrahigh energies, and result in radio synchrotron emission. (See e.g. Sarazin 2001, for a review of merger shocks and non-thermal emission from clusters.) The details of this mechanism, such as how halos and relics are induced by merging and how to accelerate the pool of non-thermal charged particles, are unclear. Nevertheless, observations continue to accumulate data, and our understanding of these sources also grows with the data base. Many interesting phenomena associated with individual sources have been found in newer observations. Among these are the imaging observations of the Mpc-scaled relics such as those found in A3667 (Röttgering et al. 1997) and A2256 (Miller et al. 2003, and references therein).

To understand how the polarized radiation interacts with intra-cluster media, we have developed a polarized radiative transfer algorithm which calculates the emission from the halos, relics or active galaxies in the clusters. We now briefly discuss the algorithm and show some results of our calculations.

\section{Polarized Radiative Transfer in Cluster Environments}

Radio sources in galaxy clusters could be halos, relics or resident active galaxies. The clusters have very tangled magnetic fields (e.g. Dolag 2001; Carilli \& Taylor 2002) which may alter the properties of the radio radiation from these sources significantly. In particular, de-polarization and Faraday rotation/mixing are important (Burn 1966; Tribble 1991), and we need to take into account these effects when we interpret the fine features in spectral-polarization imaging observations (such as that seen in clusters like A3667 and A2256).

The polarized radiative transfer equation reads

$$
\hat{D} I_{\alpha}=-\kappa_{\alpha \beta} I_{\beta}+\epsilon_{\alpha}
$$

where $I_{\alpha}=(I, Q, U, V)$ are the four Stokes parameters, $\hat{D}=c^{-1}(\partial / \partial t)+\hat{k} \cdot \nabla$ is the time and spatial differential operator, $\epsilon_{\alpha}$ are emission coefficients and $\kappa_{\alpha \beta}$ is the transfer matrix. (Here $c$ is the speed of light and $\hat{k}$ is the unit propagation vector of the light ray.) The anti-Hermitian part of $\kappa_{\alpha \beta}$ contains the absorption coefficients, and the Hermitian part contains the Faraday rotation/mixing coefficients (see Pacholczyk 1977).

In our calculations, we divide the cluster into many small cells with constant density, constant magnetic field and an homogeneous electron energy distribution. The radiative transport in each cell is determined by the equation above. We use a ray-tracing algorithm, which solves the transfer equation in the cells in succession along the rays. The resulting Stokes parameters are then used to construct the spectral-polarization images of the cluster.

For the study of the intra-cluster medium with random magnetic fields (such as those with a power-law spectrum in their distribution) or with embedded sources with complex structures on different scales, very fine cell sizes 
are needed in order to achieve good accuracy. This demands a computational algorithm which can solve efficiently the transfer equation, in which the four Stokes parameters are coupled. Instead of directly integrating the equation we seek a semi-analytic approach. By means of the transformations $I_{\alpha}^{\prime}=\Lambda_{\alpha \sigma} I_{\sigma}$, $\epsilon_{\alpha}^{\prime}=\Lambda_{\alpha \sigma} \epsilon_{\sigma}$ and $\kappa_{\alpha \beta}^{\prime}=\left(\Lambda_{\alpha \sigma} \kappa_{\sigma \tau} \Lambda_{\tau \beta}^{-1}\right)$ we obtain another radiative transfer equation

$$
\hat{D} I_{\alpha}^{\prime}=-\kappa_{\alpha \beta}^{\prime} I_{\beta}^{\prime}+\epsilon_{\alpha}^{\prime} .
$$

We diagonalize $\kappa_{\alpha \beta}^{\prime}$ by choosing a suitable $\Lambda_{\alpha \sigma}$ and obtain four decoupled differential equations, each corresponding to a component in $I_{\alpha}^{\prime}$. These equations have analytic solutions. We now just need to solve for $I^{\prime}$ using a set of complex algebraic equations. After carrying out an inverse transform of $I_{\alpha}^{\prime}$ we obtain the four Stokes parameters that describe the polarization and the spectrum of the radiation.
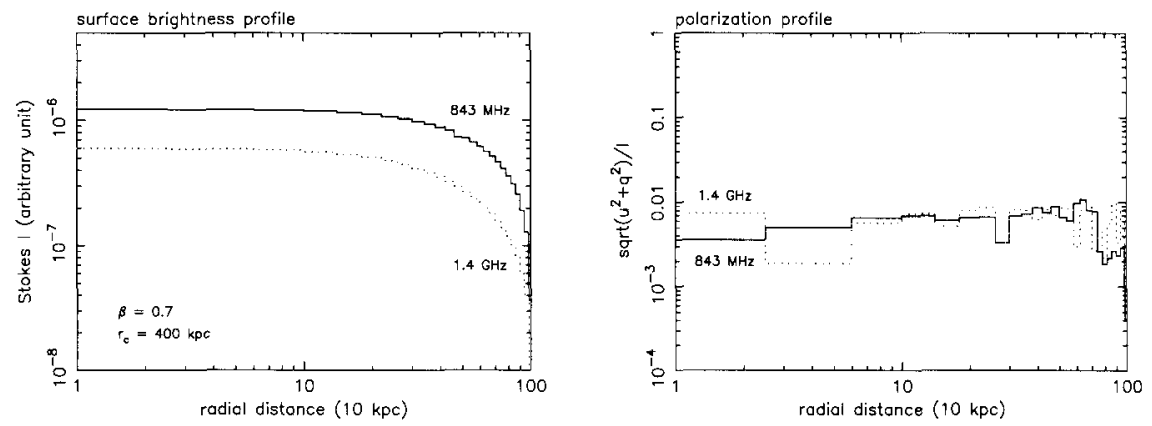

Figure 1. Surface brightness (left) and linear polarization (right) profiles of a model cluster at $843 \mathrm{MHz}$ and $1.4 \mathrm{GHz}$. The cluster parameters are $\beta=0.7$ for the density profile and core radius of $400 \mathrm{kpc}$. The energy spectral index of the electrons is 0.7. The absorption and Faraday coefficients are adopted from Jones and O'Dell (1977).

Figure 1 shows an example of a surface brightness profile and a polarization profile of a model cluster at $843 \mathrm{MHz}$ and $1.4 \mathrm{GHz}$ obtained from the calculations. The model cluster has a $\beta$ density profile and it is permeated by a random magnetic field with a flat (approximately white noise) distribution. We note that existing calculations of polarized radiation from magnetized intra-cluster media simply sum the local contribution of the four Stokes parameters, which is a good approximation when the medium is optically thin to absorption and Faraday rotation/mixing. The algorithm that we use takes full account of transfer effects, thus allowing us to calculate emission from a clumpy medium with embedded sources, which could have opacities many orders of magnitude greater than the medium.

\section{Mpc-scaled Relics: A3667, a well studied case}

As mentioned in Section 1, relics and halos are generally rare; to date some 30 to 40 cases are known. Unfortunately, most of these diffuse radio sources have not 
been studied in the detail required to obtain a deep insight into their structure and origin. Present generation telescope arrays do not have the surface brightness sensitivity to record accurately their full extent and polarization structure. An exception is A3667 which is both bright and highly polarized and has been studied in some detail.

A3667 is a rich, X-ray luminous galaxy cluster at $z=0.055$ (Sodré et al. 1992). The ROSAT X-ray image reveals that it has a slightly elongated shape (see Röttgering et al. 1997). The cold gas front at the center (Vikhlinin et al. 2001) suggests that the cluster was disturbed quite recently. The NW region of the cluster was mosaic imaged at 1.4 and $2.4 \mathrm{GHz}$ using the Australia Telescope Compact Array (ATCA) by Röttgering et al. (1997), and the rest of the region by Johnston-Hollitt (2003). The cluster was also imaged at 843 $\mathrm{MHz}$ with the Molonglo Observatory Synthesis Telescope (MOST) of Sydney University. In both the MOST and ATCA images for the entire cluster region, two remarkable arc-like diffuse radio regions are clearly visible. In addition, low resolution images reveal that there might also be a small central halo (JohnstonHollitt 2003).

The average spectral index of the northern radio arc is $\alpha=-1.0 \pm 0.2$, where $S_{\nu} \propto \nu^{\alpha}$. There is a moderate spectral-index gradient across the arc, with the flattest spectrum appearing toward the very north where the emission is most diffuse. The southern arc shows a more prominent spectral-index gradient along the major axis of A3667, with the flattest spectra at the outermost parts of the arc.

The emission from the arcs is strongly linear polarized at both 1.4 and 2.4 $\mathrm{GHz}$. The percentage polarization of the northern arc varies from 10 to $40 \%$, and the polarization appears correlated with the brightest filaments. The percentage polarization of the southern arc is at the $30-50 \%$ level. The polarization angles show complex spatial patterns, but there is no obvious correlation with the spatial distribution of the intensity. More detailed discussions of the observations, analysis, results and interpretation can be found in Johnston-Hollitt (2003) and Johnston-Hollitt et al. (2003, in preparation).

The combination of these detailed data, particularly the radio polarimetry, make A3667 an ideal platform for exploring polarized radiative transfer and testing the new code described in Section 2.

\section{References}

Buote, D. A. 2001, ApJ, 553, L15

Burn, B. J. 1966, MNRAS, 133, 67

Colafrancesco, S. 1999, in Proc. Workshop Diffuse Thermal and Relativistic Plasma in Galaxy Clusters, ed. H. Böhringer et al. (Garching: MPE), 269

Carilli, C. L., \& Taylor, G. B. 2002, ARA\&A, 40, 319

Dolag, K. 2001, in Proceedings of XXI Moriond Conference: Galaxy Clusters and the High Redshift Universe Observed in X-rays, ed. D. Neumann \& J. T. T. Van

Giovannini, G., \& Feretti, L. 2002a, in Merging Processes in Galaxy Clusters, ed. L. Ferretti et al. (Dordrecht: Kluwer), 197

Giovannini, G., \& Feretti, L. 2002b, HiA, 12, 513

Govoni, F., Ensslin, T. A., Feretti, L., \& Giovannini, G. 2001, A\&A, 369, 441 
Johnston-Hollitt, M. 2003, PhD Thesis, University of Adelaide

Jones, T. W., \& O’Dell, S. L. 1977, ApJ, 214, 522

Liang, H., Hunstead, R. W., Birkinshaw, M., \& Andreani, P. 2000, ApJ, 544, 686

Miller, N. A., Owen, F. N., \& Hill, J. H. 2003, AJ, 125, 2393

Pacholcyzk, A. G. 1977, Radio Galaxies (Oxford: Pergamon)

Röttgering, H. J. A., Wieringa, M. H., Hunstead, R. W., \& Ekers, R. D. 1997, MNRAS, 290,577

Sarazin, C. L. 2001, in Proceedings of XXI Moriond Conference: Galaxy Clusters and the High Redshift Universe Observed in X-rays, ed. D. Neumann \& J. T. T. Van (astro-ph/0105458)

Sodré, L., Capelato, H. V., Steiner, J. E., Proust, D., \& Mazure, A. 1992, MNRAS, 259,233

Tribble, P. C. 1991, MNRAS, 253, 147

Vikhlinin, A., Markevitch, M., \& Murray, S. S. 2001, ApJ, 551, 160 